\title{
Burden of Non-cardiac Patients on the Emergency Room of a Rural Cardiac Center in Sindh, Pakistan
}

\author{
Jawaid A. Sial $^{1}$, Naveedullah Khan ${ }^{1}$, Waheed Murad ${ }^{2}$, Musa Karim ${ }^{3}$
}

1. Cardiology, National Institute of Cardiovascular Diseases (NICVD), Karachi, PAK 2. Cardiology, Sheikh Khalifa Medical City, Abu Dhabi, ARE 3. Research, National Institute of Cardiovascular Diseases (NICVD), Karachi, PAK

$\square$ Corresponding author: Musa Karim, mkarim.nicvd@gmail.com

Disclosures can be found in Additional Information at the end of the article

\section{Abstract}

\section{Introduction}

The number of cardiac patients increases on a daily basis, and emergency departments bear much of the burden of non-cardiac patients due to pathological fears of the aftermath of the disease. Therefore, this study aimed to determine the burden of non-cardiac patients on the emergency department of a cardiac center in a rural area of Sindh, Pakistan.

\section{Methods}

This cross-sectional study was conducted at the emergency department of Chandka Medical College Hospital in Larkana. Consecutive patients who presented with cardiac symptoms with no previous history of cardiac disease were included. After a brief history, physical examination, electrocardiogram, and a cardiac enzyme assessment, patients were categorized as cardiac or non-cardiac. Data were analyzed using IBM SPSS Statistics for Windows, Version 21.0. (IBM Corp., Armonk, NY, US) and $\mathrm{p} \leqslant 0.05$ was statistically significant.

\section{Results}

Of the 204 patients included, 112 (59.8\%) were men, and the mean age was $47 \pm 16$ years. Most patients $(n=146 ; 71.6 \%)$ were diagnosed as non-cardiac. The non-cardiac diagnosis was significantly more common among patients without diabetes $(n=123,77.4 \%$ vs. $n=23,51.1 \%$; $=0.001)$, without chest pains $(n=93,81.6 \%$ vs. $n=53,58.9 \% ; p<0.001)$, and without shortness of breath ( $n=107,75.9 \%$ vs. $n=39,61.9 \% ; p=0.041)$.

Received 08/28/2018 Review began $08 / 31 / 2018$ Review ended 09/11/2018 Published 09/12/2018

\section{(c) Copyright 2018}

Sial et al. This is an open access article distributed under the terms of the Creative Commons Attribution License CC-BY 3.0., which permits unrestricted use, distribution, and reproduction in any medium, provided the original author and source are credited.

\section{Conclusion}

More than two-thirds of the patients were found to have a non-cardiac mechanism behind their symptoms. A major proportion of the emergency room's cardiology department is occupied by non-cardiac patients. Owing to its direct and indirect implication on an otherwise struggling health system, we suggest chest pain units should be developed to decrease the workload and provide better care to cardiac patients.

Categories: Cardiology, Public Health

Keywords: chest pain, cardiac, non-cardiac, emergency, burden 


\section{Introduction}

According to the Institute of Health Metrics and Evaluation profile of Pakistan, ischemic heart disease (IHD) and cerebrovascular disease were the leading causes of death in 2016, with a percentage increase of $23.0 \%$ and $13.4 \%$ from 2005 to 2016 , respectively. IHD was also the leading cause of premature deaths, with a percentage increase of 21.4\% from 2005 to 2016 [1]. With over 207 million inhabitants, Pakistan has endured a major portion of the global burden of deaths due to cardiovascular diseases (CVDs) [2]. Among other environmental and heritable factors that aggravate the prevalence and subsequent mortality due to CVD, overcrowding emergency departments in an already limited health care system in Pakistan is a serious problem for reliable health care [3-6].

Recent studies have declared the state of emergency care in Pakistan as sub-optimal, with deficiencies in both human resources as well as the availability of equipment and levels of supplies $[4,7]$. In such a resource-limited health care system, the degree of burden of noncardiac patients on a cardiac emergency department is worrisome. Chest pain is the leading precipitant of cardiac emergency department visits all over the world [8-11]. Chest pain categorized as non-cardiac in origin accounts for around $45 \%$ to $77 \%$ of the patients presenting to emergency departments [10,12-18]. This substantially high frequency of non-cardiac chest pain can be attributed to the increasing awareness among the general population regarding the lethal manifestation and pathological fears of the aftermath of heart disease. This pathological fear is termed cardiophobia and is prevalent among nearly half of the patients with a noncardiac chest pain [13,19-20].

The most commonly found underlying mechanisms of non-cardiac chest pain reported in past studies are gastroesophageal reflux disorder (GERD), esophageal hypersensitivity and dysmotility, musculoskeletal pain, major depressive disorder (MDD), and pericarditis [8,13,21].

Non-cardiac chest pain is not only a financial burden on the health care system but also results in the inappropriate use of health care facilities [10]. A study in the United States estimated the cost of the initial care of patients ultimately diagnosed with non-cardiac disease is around eight billion dollars [15]. In countries like Pakistan, where the health care provider-to-seeker ratio is already imbalanced, non-cardiac chest pain is a major concern, with both direct and indirect impacts on the health care system, in terms of health care cost and utilization. Therefore, the aim of this study was to determine the burden of non-cardiac patients on the emergency department of a cardiac center in the rural areas of Sindh, Pakistan.

\section{Materials And Methods}

This cross-sectional study was conducted at the emergency department of Chandka Medical College, Larkana, from January 15, 2015, to April 14, 2015. After receiving approval of the study design from our institutional ethical review committee, we conducted a review of the consecutive patients who presented to the emergency department with concerns of chest pain, shortness of breath (SOB), palpitation, or syncope. The importance and befits of the study were explained, and we obtained informed consent from all enrolled patients or their legal caretakers. Patients with a prior history of acute coronary syndrome (ACS) or congenital heart disease were excluded. We reviewed patient demographic details, such as age, gender, relevant medical history (e.g., family history of premature coronary artery diseases, personal history of hypertension, smoking, and diabetes mellitus), and presenting concerns such as chest pain, SOB, palpitation, and syncope. After a brief history and examination, electrocardiographic assessment, and cardiac enzyme test (where needed), patients were categorized as "cardiac" or "non-cardiac." To avoid the observational bias, patient reports were assessed by three independent cardiologists with more than five years' experience, each blinded to the diagnosis by the remaining two, and patients were classified as cardiac or non-cardiac cases based on an 
agreement of at least two of the cardiologists. The sample size for the study was calculated with an expected prevalence of non-cardiac symptoms as 55\% [14], a 95\% confidence level, and a margin of error of 7\%. Based on these assumptions, the calculated sample size was 195 patients; we recruited 10 additional patients to ensure set precision in the event of information loss. Of the 205 patients enrolled, one patient was excluded from the analysis due to missing information on the required variables.

Data were collected on predesigned structural proforma. Collected data were entered and analyzed using IBM SPSS Statistics for Windows, Version 21.0. (IBM Corp., Armonk, NY, US). Categorical variables were expressed as frequency and percentage. Minimum, maximum, and mean \pm standard deviation (SD) were calculated for continuous variables. The chi-square test was performed to assess the outcome by demographic and baseline characteristics. Multivariate logistic regression was applied to assess the effect of patients' baseline characteristics on noncardiac diagnosis. A two-sided p-value of $\leqslant 0.05$ was taken as criteria for statistical significance.

\section{Results}

Of the 204 included patients, 112 (59.8\%) were men, and the mean age $( \pm$ SD) of the patients was $47 \pm 16$ years with a minimum age of 14 years and a maximum age of 90 years. Most patients $(n=129,63.2 \%)$ were 50 years or younger. More than half of the patients $(n=112$, $54.9 \%$ ) were hypertensive. Seventeen patients (8.3\%) had a family history of premature coronary artery diseases (CAD). Ninety-nine patients (44.1\%) presented to the emergency department with chest pain while SOB was the second most common presenting concern. Clinical history and presenting concerns are presented in Table 1. 


\section{Cureus}

\section{Baseline Characteristics}

\section{Gender}

Male

Female

Age [Mean $\pm S D$ ]

Up to 50 years

More than 50 years

\section{Clinical History}

Family History CAD

Hypertension

Diabetes Mellitus

Smoking

Presenting Concern

Chest Pain

Shortness of Breath

Palpitation

Syncope

\section{Frequency (\%)}

$122(59.8 \%)$

$82(40.2 \%)$

$47.33 \pm 15.83$ years

$129(63.2 \%)$

75 (36.8\%)

$17(8.3 \%)$

$112(54.9 \%)$

$45(22.1 \%)$

$31(15.2 \%)$

$90(44.1 \%)$

$63(30.9 \%)$

$21(10.3 \%)$

$2(1 \%)$

\section{TABLE 1: Clinical history and presenting concerns}

Abbreviations: SD, Standard Deviation; CAD, Coronary Artery Diseases

One hundred forty-six patients (71.6\%) had a non-cardiac mechanism behind their symptoms. Cardiac abnormalities were diagnosed in 58 patients (28.4\%). Of these cardiac patients, 38 (65.5\%) had ACS, 11 (19.0\%) had valvular abnormalities, and nine (15.5\%) had heart failure. Patients without diabetes were more likely to have a non-cardiac diagnosis $(p=0.001)$. Similarly, patients who presented without chest pain or SOB and with palpitation were more likely to have a non-cardiac diagnosis $(\mathrm{p}<0.001, \mathrm{p}=0.041$, and $\mathrm{p}=0.043$, respectively). The final diagnoses, according to baseline characteristics and presenting concerns, are presented in Table 2.

\begin{tabular}{|c|c|c|c|c|}
\hline Baseline Characteristics & Base & Final Dia & & \\
\hline Frequency (row \%) & $\mathbf{N}$ & Cardiac & Non-Cardiac & \\
\hline
\end{tabular}

Gender 


\section{Cureus}

Male

Female

Age

Up to 50 years

More than 50 years

Family History CAD

Yes

No

\section{Hypertension}

Yes

No

Diabetes Mellitus

Yes

No

\section{Smoking}

Yes

No

\section{Chest Pain}

Yes

No

Shortness of Breath

Yes

No

\section{Palpitation}

Yes

No

\section{Syncope}

Yes

No
122

82

129

75

17

187

112

92

45

159

31

173

90

114

63

141

21

183

$2(9.5 \%)$

$56(30.6 \%)$

2

202

$33(27 \%)$

$25(30.5 \%)$

$22(29.3 \%)$

$3(17.6 \%)$

55 (29.4\%)

$32(28.6 \%)$
$89(73 \%)$

0.593

57 (69.5\%)

$93(72.1 \%)$

$53(70.7 \%)$

$14(82.4 \%)$

$132(70.6 \%)$

0.303

$80(71.4 \%)$

$26(28.3 \%) \quad 66(71.7 \%)$

$22(48.9 \%) \quad 23(51.1 \%)$

$123(77.4 \%)$

$0.001^{*}$

$21(67.7 \%)$

$48(27.7 \%) \quad 125(72.3 \%)$

$53(58.9 \%)$

$93(81.6 \%)$

$<0.001^{*}$

$0.041^{*}$

$107(75.9 \%)$

$19(90.5 \%)$

$127(69.4 \%)$

$0.043^{*}$

0.37

TABLE 2: Final diagnosis by baseline characteristics of the patients 


\section{Cureus}

Abbreviations: CAD, Coronary Artery Diseases

** P-values are based on the chi-square test

* Significant at the $5 \%$ level of significance

On a multivariate logistic regression model with a non-cardiac diagnosis as a dependent variable (Hosmer and Lemeshow test $p$-value $=0.611$ ), presenting without diabetes, chest pain, or SOB was significant $(\mathrm{p}=0.005,<0.001$, and $\mathrm{p}=0.003$, respectively; odds ratios of $2.88,4.54$, and 3.37 , respectively) while palpitation at presentation was found to be insignificant $(\mathrm{p}=$ $0.113)$.

\section{Discussion}

This study was conducted to determine the burden of non-cardiac patients on a cardiac emergency department. We found more than two-thirds (71.6\%) of the patients who visited the cardiac emergency room with concerns of chest pain, SOB, palpitation, or syncope had a noncardiac mechanism behind their symptoms. A cardiac symptom (e.g., chest pain) eventually diagnosed to be of non-cardiac origin is the pertinent problem every cardiac emergency room faces. The frequency of non-cardiac origin for symptoms in our study falls within the range of $45 \%$ to $77 \%$ reported in previous studies $[10,12-18]$. There is always uncertainty among physicians regarding discharging patients with no viable explanation for their symptoms. There is always the fear of missed diagnosis and overtreatment, which may result in life-threatening circumstances or side effects from unnecessary medication and higher health care costs [11]. In our study, the univariate and multivariate analyses revealed that the non-cardiac diagnosis among the patients who presented with cardiac symptoms was significantly higher among patients without diabetes $(\mathrm{n}=123,77.4 \%)$ compared to those with diabetes $(\mathrm{n}=23,51.1 \% ; \mathrm{p}=$ 0.001). Patients' presenting concerns have certain clues to indicate non-cardiac origins; for example, patients presenting without chest pain are 4.54 times more likely to have a noncardiac origin of their symptoms with a frequency comparison of 93 (81.6\%) vs. 53 (58.9\%), p < 0.001. Similarly, patients presenting without SOB are 3.37 times more likely to be diagnosed as non-cardiac, with a frequency compassion of 107 (75.9\%) vs. 39 (61.9\%), p = 0.041.

We did not determine the cause of symptoms for the non-cardiac patients. However, various causes have been identified over the years for non-cardiac symptoms, especially non-cardiac chest pain. The most common causes are GERD, esophageal hypersensitivity and dysmotility, musculoskeletal pain, MDD, and pericarditis [8,13,21].

The high burden of non-cardiac patients on a resource-strained cardiac emergency room in a developing country like Pakistan [4,7] is a serious issue that warrants the attention of both health care professionals and governing bodies. It is important to focus on developing chest pain units for the rapid assessment of non-cardiac causes and patient risk assessments [22]. Various efficient, cost-effective, and easily adoptable risk stratification modalities have been proposed in the literature, such as the report by Backus et al. [11], who proposed a score composed of patient history, electrocardiogram, age, risk factors, and troponin levels (HEART), for accurate decision-making and triage. Soares-Filho et al. [14] reported a significant proportion of psychiatric illness among the patients with symptoms indicative of chest pain and recommended to apply the hospital anxiety and depression scale (HADS) method for the screening of such patients. Al-Ani et al. [13] found GERD, depression, and anxiety more common than CAD in patients presenting with acute chest pain. Similarly, Hadlandsmyth et al. [15] reported a significant association of interoceptive fear and anxiety with health care utilization among patients with chest pain. 


\section{Limitations}

Our single-center study's small sample size and geographic coverage limit the generalizability of our findings; for a thorough understanding of the non-cardiac burden on the cardiac emergency room, larger multicenter studies are needed.

\section{Conclusions}

More than two-thirds of the patients were found to have a non-cardiac mechanism behind their symptoms. A major part of emergency room cardiology resources is occupied by non-cardiac patients. Owing to its direct and indirect implication on an otherwise struggling health system, we suggest chest pain units should be developed to decrease the workload and provide better care to cardiac patients.

\section{Additional Information \\ Disclosures}

Human subjects: Consent was obtained by all participants in this study. Chandka Medical College, Larkana issued approval SMBBMU/01/15. Approval was taken from the institutional ethical review committee of Chandka Medical College, Larkana, Sindh, Pakistan. Animal subjects: All authors have confirmed that this study did not involve animal subjects or tissue. Conflicts of interest: In compliance with the ICMJE uniform disclosure form, all authors declare the following: Payment/services info: All authors have declared that no financial support was received from any organization for the submitted work. Financial relationships: All authors have declared that they have no financial relationships at present or within the previous three years with any organizations that might have an interest in the submitted work. Other relationships: All authors have declared that there are no other relationships or activities that could appear to have influenced the submitted work.

\section{References}

1. The Institute for Health Metrics and Evaluation (IHME). Country profiles-Pakistan . (2016). Accessed: August 20, 2018: http://www.healthdata.org/pakistan.

2. Shakeel M, Irfan M, Khan IA: Estimating the mutational load for cardiovascular diseases in Pakistani population. PloS One. 2018, 13:e0192446. 10.1371/journal.pone.0192446

3. George F, Evridiki K: The effect of emergency department crowding on patient outcomes . Health Sci J. 2015, 9:1.

4. Razzak JA, Baqir SM, Khan UR, Heller D, Bhatti J, Hyder AA: Emergency and trauma care in Pakistan: a cross-sectional study of healthcare levels. Emerg Med J. 2015, 32:207-213. 10.1136/emermed-2013-202590

5. Sun BC, Hsia RY, Weiss RE, et al.: Effect of emergency department crowding on outcomes of admitted patients. Ann Emerg Med. 2013, 61:605-611. 10.1016/j.annemergmed.2012.10.026

6. Bernstein SL, Aronsky D, Duseja R, et al.: The effect of emergency department crowding on clinically oriented outcomes. Acad Emerg Med. 2009, 16:1. 10.1111/j.1553-2712.2008.00295.x

7. Razzak JA, Hyder AA, Akhtar T, Khan M, Khan UR: Assessing emergency medical care in low income countries: a pilot study from Pakistan. BMC Emerg Med. 2008, 8:8. 10.1186/1471227X-8-8

8. Paichadze N, Afzal B, Zia N, Mujeeb R, Khan MM, Razzak JA: Characteristics of chest pain and its acute management in a low-middle income country: analysis of emergency department surveillance data from Pakistan. BMC Emerg Med. 2015, 15:S13. 10.1186/1471-227X-15-S2S13

9. Gerber TC, Kontos MC, Kantor B: Emergency department assessment of acute-onset chest pain: contemporary approaches and their consequences. Mayo Clin Proc. 2010, 85:309-313. 10.4065/mcp.2010.0141

10. McDevitt-Petrovic O, Kirby K, Shevlin M: The prevalence of non-cardiac chest pain (NCCP) using emergency department (ED) data: a Northern Ireland based study. BMC Health Serv Res. 
2017, 17:549. 10.1186/s12913-017-2493-8

11. Backus BE, Six AJ, Kelder JC, et al.: Chest pain in the emergency room: a multicenter validation of the HEART score. Crit Pathw Cardiol. 2010, 9:164-169.

10.1097/HPC.0b013e3181ec36d8

12. Bhuiya FA, Pitts SR, McCaig LF: Emergency department visits for chest pain and abdominal pain: United States, 1999-2008. NCHS Data Brief. 2010, 1-8.

13. Al-Ani M, Winchester DE: Prevalence and overlap of noncardiac conditions in the evaluation of low-risk acute chest pain patients. Crit Pathw Cardiol. 2015, 14:97-107. 10.1097/HPC.0000000000000050

14. Soares-Filho GL, Freire RC, Biancha K, Pacheco T, Volschan A, Valença AM, Nardi AE: Use of the hospital anxiety and depression scale (HADS) in a cardiac emergency room: chest pain unit. Clinics (Sao Paulo). 2009, 64:209-214. 10.1590/S1807-59322009000300011

15. Hadlandsmyth K, Rosenbaum DL, Craft JM, Gervino EV, White KS: Health care utilisation in patients with non-cardiac chest pain: a longitudinal analysis of chest pain, anxiety and interoceptive fear. Psychol Health. 2013, 28:849-861. 10.1080/08870446.2012.762100

16. Lee TH, Goldman L: Evaluation of the patient with acute chest pain. N Engl J Med. 2000, 342:1187-1195. 10.1056/NEJM200004203421607

17. Bozkurt Zincir S, Sunbul M, Aydin Sunbul E, Dalkilic B, Cengiz F, Kivrak T, Durmus E: Evaluation of alexithymia, somatosensory sensitivity, and health anxiety levels in patients with noncardiac chest pain. Biomed Res Int. 2014, 2014:896183. 10.1155/2014/896183

18. Dumville JC, MacPherson H, Griffith K, Miles JN, Lewin RJ: Non-cardiac chest pain: a retrospective cohort study of patients who attended a rapid access chest pain clinic. Fam Pract. 2007, 24:152-157. 10.1093/fampra/cmm002

19. Eifert GH: Cardiophobia a paradigmatic behavioural model of heart-focused anxiety and nonanginal chest pain. Behav Res Ther. 1992, 30:329-345. 10.1016/0005-7967(92)90045-I

20. Zvolensky MJ, Feldner MT, Eifert GH, Vujanovic AA, Solomon SE: Cardiophobia: a critical analysis. Transcult Psychiatry. 2008, 45:230-252. 10.1177/1363461508089766

21. Fass R, Achem SR: Noncardiac chest pain: epidemiology, natural course and pathogenesis . J Neurogastroenterol Motil. 2011, 17:110-123. 10.5056/jnm.2011.17.2.110

22. Kontos MC, Diercks DB, Kirk JD: Emergency department and office-based evaluation of patients with chest pain. Mayo Clin Proc. 2010, 85:284-299. 10.4065/mcp.2009.0560 